\title{
Skin-sparing mastectomy in breast cancer
}

\section{Patricia Villarreal-Colin*1, Carlos Robles-Vidal ${ }^{1}$ and Monica Drucker- Zertuche $^{2}$}

\author{
Address: ${ }^{1}$ Department of Breast Tumors, Instituto Nacional de Cancerología, Mexico and ${ }^{2}$ Plastic Surgery Service, Instituto Nacional de \\ Cancerología, Mexico \\ Email: Patricia Villarreal-Colin* - patyvics@gmail.com \\ * Corresponding author
}

from $24^{\text {th }}$ Annual Meeting of the National Cancer Institute of Mexico

Mexico City, Mexico. 14-17 February 2007

Published: 5 February 2007

BMC Cancer 2007, 7(Suppl I):A48 doi:10.I I86/I47I-2407-7-SI-A48

This article is available from: http://www.biomedcentral.com/I47I-2407/7/SI/A48

(C) 2007 Villarreal-Colin et al; licensee BioMed Central Ltd.

\section{Background}

Breast cancer is one of the main health problems in women in Mexico. Breast cancer treatment needs to be multidisciplinary. Surgical treatment has presented dramatics changes. Skin-sparing mastectomy (SSM) greatly enhances the aesthetic results of breast reconstruction, is not yet a standard technique for breast cancer treatment, although it is shown as an advanced and promising procedure. The objective of this study was to present the experience of the National Cancer Institute of Mexico in skinsparing mastectomy in patients with breast cancer.

\section{Materials and methods}

A retrospective review was performed in patients who underwent SSM and immediate breast reconstruction from April 1997 to December 2004. Records were analyzed for each patient, including type of tumors and treatment, overall survival, disease free survival and aesthetic results.

\section{Results}

During April 1997 and December 2004, SSM with immediate reconstruction was performed in 91 patients (4 patients with bilateral cancer and 10 underwent prophylactic mastectomy in the other breast). The mean age was 40 years (22-58 years). Most patients were pre-menopausal $(77 \%)$. Five patients (5.5\%) have contralateral breast cancer. The histological diagnoses was invasive ductal carcinoma in the most patients (74.7\%). Systemic neoadjuvant chemotherapy was performed in $32.9 \%$ (30 patients) and radiotherapy preoperative in $7.7 \%$ (7 patients). Most of half of patient (58) underwent adjuvant chemotherapy $(63.3 \%)$ and 27 patients received adjuvant radiotherapy (29.6\%). In most of the patients (50) TRAM flap was the reconstruction method employed, tissue expanderimplant in second place (27 patients), implant in 23 patients and latissimus flap and implant in 5 patients. The complication rate was 36\% (38 cases in 105 procedures), 28 minor and 9 majors. Of 7 patients with preoperative radiotherapy 6 underwent complications (3 majors) and 11 of 27 patients with adjuvant radiotherapy have any type of complication (4 majors). The aesthetic result was in $63.3 \%$ this was excellent or good; $14.4 \%$ regular; $7.7 \%$ poor and $14.4 \%$ not reported. The follow-up mean was 66 months (13-100 months), four recurrences were detected (4.4\%); 3 systemic and 1 local-regional and systemic.

\section{Conclusion}

SSM is an oncological safety procedure in breast cancer early with recurrence rates similar to non-SSM, the complication rates were comparable to conventional mastectomy, being mostly associated to radiotherapy. 